\title{
Computerbasiertes anonymes Critical Incident Reporting: ein Beitrag zur Patientensicherheit
}

\author{
M. Kaufmann ${ }^{a}$, S. Staender ${ }^{b}$, G. von Below ${ }^{c}$, H. H. Brunner ${ }^{c}$, L. Portenier ${ }^{\text {, D. D. Scheidegger }}{ }^{a}$
}

La version française suivra.

a Dep. Anästhesie, UHBS Basel

b Abt. Anästhesie, Spital Männedorf

c Verbindung der Schweizer Ärztinnen und Ärzte FMH

d Schweizer Berufsverband der Krankenschwestern und Krankenpfleger SBK-ASI

Korrespondenz:

PD Dr M. Kaufmann

Departement Anästhesie

KBS, Universitätskliniken

CH-4031 Basel

Interessensbindungen:

Es bestehen keinerlei Interessensverbindungen, die Arbeit wurde unterstützt durch die Perioperative Patient Safety Group des Dep. Anästhesie, KBS Universitätskliniken, Basel

\section{Zusammenfassung}

In der Vergangenheit wurde der Patientensicherheit als wesentliches Qualitätsmerkmal eines Behandlungsprozesses nicht die notwendige Beachtung geschenkt. Ein kritisches Element zur Erhöhung der Sicherheit ist die Schaffung einer Sicherheitskultur mit dem Effekt, dass Fehler identifiziert, Ursachen abgeklärt und darauf basierend Massnahmen zur künftigen Verhinderung dieser Fehler ergriffen werden. Erfahrungen aus verschiedenen Gebieten haben gezeigt, dass das systematische Erfassen von kritischen Ereignissen (Critical Incident Reporting) ein wichtiges Element einer solchen Sicherheits- und Lernkultur darstellt. Anonyme, freiwillige Meldesysteme haben im Gegensatz zu obligatorischen Meldesystemen das Potential, auf einer breiten Basis Systemschwächen und Fehler aufzuzeigen, bevor daraus gravierende Komplikationen entstehen.

Der folgende Artikel stellt ein System vor, das breit abgestützt (Ärzte, Pflege, FMH, Apotheker) auf der Basis der im Bereich Anästhesie seit vielen Jahren betriebenen Critical-Incident-Reporting-Systeme (CIRS) entstanden ist. Dieses generalisierte CIRSmedical-System (PC-, Intranet- oder Internet-basiert) definiert indirekt einen minimalen Datensatz, behält aber eine hohe fachund berufsgruppenspezifische Konfigurierbarkeit. Das System wird interessierten Kreisen gratis zur Verfügung gestellt (www.CIRSmedical.org) und soll dazu dienen, die Schwelle zur Einführung solcher Systeme zu senken. Es kann lokal, dezentral in Spitälern und auch übergreifend in geschlossenen Benutzergruppen eingesetzt werden.

\section{Einleitung}

Patientensicherheit ist ein wichtiges Anliegen in heutigen Gesundheitssystemen. Es ist in den letzten Jahren sichtbar geworden, dass viele Patienten im Rahmen der routinemässigen medizinischen Betreuung unbeabsichtigt zu Schaden kommen [1,2]. Daten aus verschiedenen Ländern deuten darauf hin, dass, obwohl die Mehrzahl dieser sogenannten Adverse Events ohne bleibende Konsequenzen sind, bei etwa $1 \%$ der Patienten mit relevanten Schäden (bis hin zum Tode) zu rechnen ist. Von Bedeutung ist, dass beinahe die Hälfte dieser Zwischenfälle (Adverse Events) meist auf der Basis von kritischen Ereignissen (Critical Incidents) grundsätzlich vermeidbar wäre [3]. Wenn man bedenkt, dass ein grösseres Spital mehr als 100000 Patienten pro Jahr ambulant und stationär behandelt, wird sichtbar, wie relevant dieses Problem in Tat und Wahrheit ist und welche Kostenfolgen (Verlängerung Spitalaufenthalt, bleibende Morbidität, reduzierte Erwerbsfähigkeit, Haftpflicht, ...) für das Gesundheitswesen und die Gesellschaft daraus resultieren.

Solche einzelne Zwischenfälle werden nur allzu häufig als Fehlhandlung oder Versagen von einzelnen dargestellt («human error»). Analysen von Critical Incidents wie auch Erfahrungen aus der Fliegerei, der Nuklear- und Ölindustrie oder anderen komplexen Systemen haben aber deutlich gezeigt, dass solche Ereignisse fast immer multifaktoriell verursacht sind: Arbeitsbelastung, Kommunikations-, Ausbildungs- und Überwachungsprobleme, ungenügende Ressourcen, Teamfaktoren, inadäquate Umgebung und last but not least Patientenfaktoren sind meist entscheidend mitbeteiligt $[4,5]$. Im Schweizerischen Gesundheitswesen sind präventive Strategien zur Beeinflussung von solchen Aspekten im Gegensatz zu klareren Strategien im Ausland noch unterentwickelt (Tab. 1). So waren es in der Vergangenheit hauptsächlich medizinische Fachdisziplinen mit einem ausgeprägten Sicherheitsverständnis (z.B. Anästhesiologie), die sich frühzeitig mit der Thematik befasst haben und methodische Ansätze aus anderen Sicherheitskulturen (z. B. Fliegerei) übernommen und angepasst haben $[6,7]$.

\section{Critical Incident Reporting als Basis zum «Lernen aus Fehlern»}

Unter kritischen Zwischenfällen im allgemeinen versteht man Ereignisse oder Umstände, die einen negativen (oder positiven) Einfluss auf die Ziele eines Systems haben (können). In bezug auf das Gesundheitswesen existieren viele verschiedene Definitionen [11]. Wir verstehen darunter 
Tabelle 1

Kernelemente präventiver Strategien im Ausland.

\begin{tabular}{|l|c|}
\hline GB: National Health Service (NHS) & USA: US Institute of Medicine \\
\hline - Teamorientiertes Training mit dem & - Verbreitung und Verbesserung des Wissens \\
Verständnis, dass die Beschreibung von & zu Sicherheitsaspekten \\
Zwischenfällen wichtig ist für andere & - Obligatorisches Reporting von Zwischen- \\
Teammitglieder & fällen (Adverse Events) \\
- Möglichkeit von wiederholtem Auftreten & - Definieren und Vorgeben von Standards \\
von kritischen Ereignissen wird erkannt & durch Organisationen und Fach- \\
- Berichterstattung ohne Schuldzuweisung & gesellschaften \\
- Koordinierte Ereignis-Datenbank und & - Einführung von auf Sicherheit ausgerich- \\
verantwortungsvoller Umgang mit den & teten Prozessen \\
Daten & \\
- Feedback an die Berichter von kritischen & \\
Ereignissen & \\
- Aktives Lernen & \\
- Systemisch-präventiver Ansatz & \\
- Risk-Management-Bewusstsein & \\
\hline
\end{tabular}

Ereignisse, die ohne Intervention zu einem unerwünschten Ausgang, d.h. einer physischen oder psychischen Beeinträchtigung eines Patienten, hätte führen können (oder trotz Intervention dazu geführt haben); d.h. Critical Incidents haben primär einen verminderten «Margin of Safety-» und nicht einen Komplikations- respektive Adverse-Event-Charakter.

Schwere Zwischenfälle und Komplikationen (Adverse Events) sind relativ selten. Es macht nur bedingt Sinn, die Systemsicherheit durch die akribische Analyse von solchen seltenen Einzelereignissen zu verbessern, wenn tagtäglich auch bei der Arbeit im Gesundheitswesen Vorstufen zu Adverse Events, sogenannte Critical Incidents (CI) passieren. Solche CI haben das Potential, unter bestimmten Umgebungsbedingungen $\mathrm{zu}$ einer Komplikation auszuufern. Durch die hohe Frequenz von CI im klinischen Alltag stellt deren Untersuchung eine viel grössere und fundiertere

\section{Abbildung 1}

Das «Swiss Cheese Modell» zu systembasierten Adverse Events: Moderne Systeme haben in ihren Prozessen meist Sicherheitsbarrieren (Alarmsysteme, Vorschriften, Kontrollen, Standards, physikalische Vorkehrungen, usw.) eingebaut. Solche Barrieren sind aber nie perfekt. Selten sind diese Lücken so ausgerichtet, dass eine unglückliche Verkettung von Ereignissen (chain of events) zu einem Adverse Event führt. Kritische Ereignisse haben das Potential, Lücken in den verschiedenen Sicherheitsbarrieren aufzuzeigen.

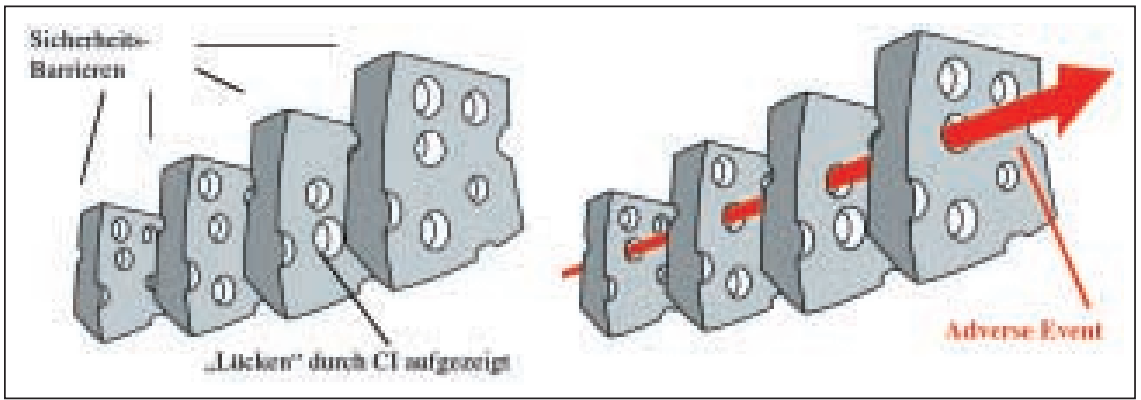

Basis für Bemühungen zur Erhöhung der Systemsicherheit dar. Solche Incidents decken kleine und grosse Sicherheitslücken im System auf, deren Schliessung zur Erhöhung der Gesamtsicherheit beiträgt. Die Critical-Incident-Technik zeichnet sich dadurch aus, dass durch das Sammeln von Informationen über kritische Zwischenfälle Erkenntnisse für Korrekturen ( «Schliessen von Sicherheitslücken») gewonnen werden können und damit künftige Fehler (Adverse Events) vermieden werden [8, 9] (Abb. 1).

Dass Incident Reporting auch in der Schweiz ein zentrales Element von Risk-Management darstellt, wurde im Rahmen der Task Force «TOWARDS A SAFE HEALTHCARE SYSTEM, proposal for a national programme on patient safety improvement for Switzerland» festgehalten (Luzern 4/2001) [10].

\section{Vorteile von Critical Incident Reporting}

\section{$[7,9,11]$}

- Kritische Zwischenfälle werden in der Regel kurzfristig durch die direkt Involvierten an der Front gut memoriert.

- Eine Sammlung von solchen Ereignissen kann strukturiert untersucht werden, Folgerungen daraus können $u$.a. auch verwendet werden, um die positiven Faktoren eines Systems zu verstärken.

- Diese Technik ist günstig im Betrieb und hat einen hohen Informationsgehalt.

- Diese Technik betont Elemente, die ein System besonders verletzbar machen.

- Diese Technik ist besonders geeignet für seltene, atypische Ereignisse.

- Es eignet sich auch als Sammelgefäss für das Reporting von Near-Misses, wenn dies auch nicht die primäre Zielrichtung eines CITbasierten Systems ist.

- Diese Technik ermöglicht Einsicht in Bewältigungs- und Vermeidungsstrategien.

\section{Nachteile von Critical Incident Reporting}

- Ein Problem liegt darin, dass nur Ereignisse, die bewusst realisiert und memoriert werden, in ein solches System eingehen können.

- Ein exaktes und wahrheitsgetreues Reporting ist eine weitere Voraussetzung.

- Die CIT-Technik betont Ereignisse, die eher selten sind, während sehr häufige, «banale» Ereignisse eher untergehen. Grundsätzlich ist das «Under-reporting» in denjenigen Umgebungen ein Problem, in die diese Technik und das damit verbundene mind-set noch ungenügend im alltäglichen Arbeitsumfeld integriert werden konnte («EisbergPhänomen»). 
Tabelle 2

Rückblick auf die Geschichte des Incident Reportings und dessen WEB-basierte Umsetzung.

\begin{tabular}{ll}
1947 & Fitts \& Jones: Analyse von Pilotenfehlern \\
1965 & Hubbard: Clinical competence of residents (N Engl J Med) \\
\hline 1987 & Runciman: Australian Incident Monitoring study in Anaesthesia \\
\hline 1995 & $\begin{array}{l}\text { INTERNET + Incident Reporting in Anesthesia } \rightarrow \text { CIRS }^{\circ} \text { worldwide } \\
\text { (DA, Uni Basel: www.anaesthesie.ch/cirs/) }\end{array}$ \\
\hline 1998 & CIRS $^{\odot}+$ Fachgesellschaft SGAR $\rightarrow$ CIRS-CH (SGAR: www.sgar-ssar.ch/qualitaet/) \\
\hline 2002 & CIRS $^{\odot}+$ FMH / SBK $=>$ CIRSmedical ${ }^{\odot}$ (POPS-UHBS, FMH, SBK: www.CIRSmedical.org)
\end{tabular}

\section{Umsetzung}

Um ein einheitliches Reporting von kritischen Ereignissen im Gesundheitswesen über alle Fachdisziplinen hinweg zu fördern, hat die Perioperative Patient Safety Group der Universitätskliniken Basel (UHBS) auf der Basis ihrer langjährigen Erfahrung (Tab. 2) in Zusammenarbeit mit FMH, GSASA, UNI Basel und Pflege (SBK) den minimalen Datensatz eines generischen anonymen kritischen Incident-Systems definiert, unter www.CIRSmedical.ORG beispielhaft zugänglich gemacht und verschiedensten Institutionen bereits zur Verfügung gestellt. Dieses 4sprachige System (d/f/i/e) zeichnet sich dadurch aus, dass es durch einen auf das Wesentliche reduzierten minimalen Datensatz die

\section{Abbildung 2}

Struktur des elektronischen Eingabeformulars. Im Abschnitt «Ereignis» werden je nach gewählter Berufsgruppe und dem Verantwortungsbereich (Allgemeinmedizin, Chirurgie, Gynäkologie, Innere Medizin, Anästhesie, usw.) automatisch unterschiedliche, für die jeweilige Fachdisziplin passende strukturierte Kategorisierungselemente vorgegeben.

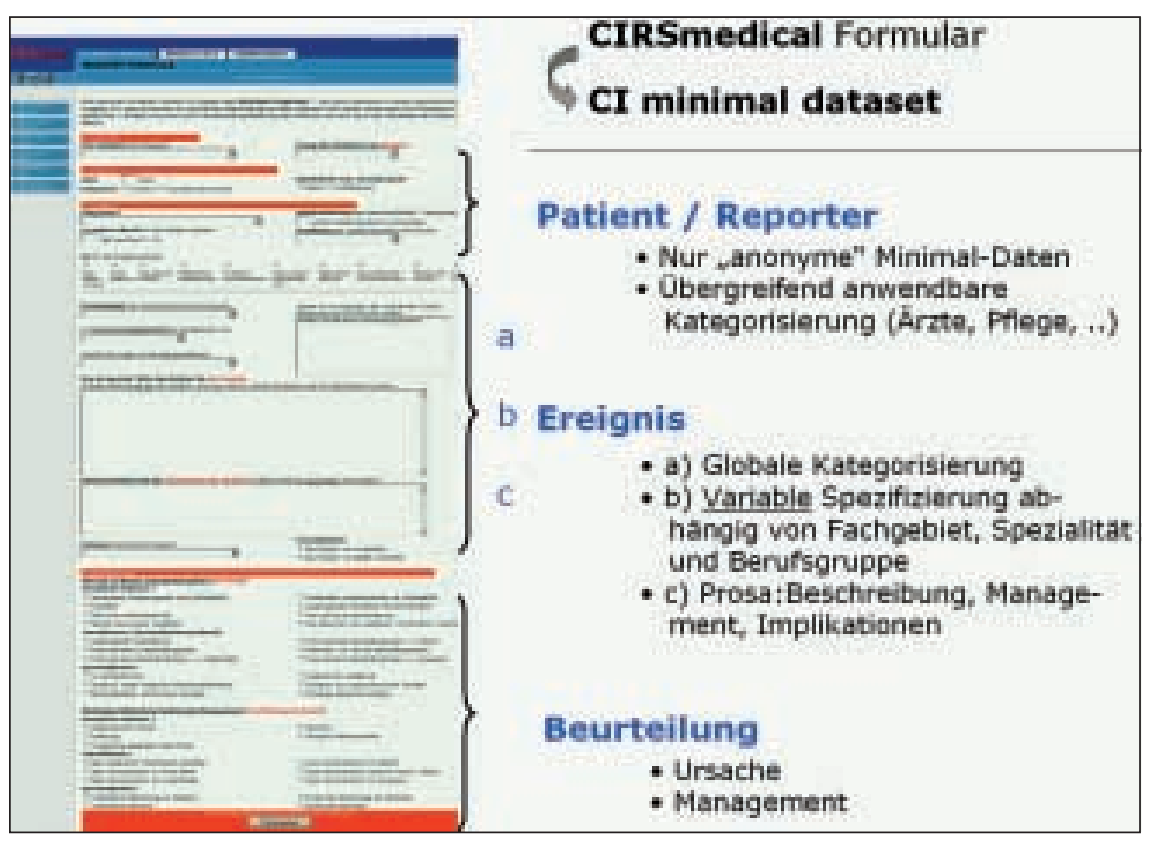

Compliance der Eingeber im Alltag erreicht und trotzdem eine hohe fach- und berufsgruppenspezifische Konfigurierbarkeit beibehält (Abb. 2):

- Reporter: Neben der Berufsgruppe wird lediglich noch der Bezug des Berichters zum Ereignis festgehalten.

- Die Angaben zum Patienten reduzieren sich auf das Alter des Patienten und die Versorgungsart (geplant vs. notfallmässig), falls überhaupt anwendbar.

- Das Ereignis wird detaillierter erfasst. Neben der Ereignisart werden Uhrzeit, Arbeitsbelastung des Verursachers, Ausbildung, Ereignisort, Berufsgruppe des Verursachers, Verantwortungsbereich (Fachdisziplin) und davon abhängig strukturiert spezifische Zusatzelemente festgehalten: Einem ärztlichen Verursacher aus dem Fach Gynäkologie-Geburtshilfe werden beispielsweise zu den Themenbereichen Gynäkologie, Ambulatorium, OP/ Anästhesie, Endokrinologie, Präpartalstation, Gebärsaal, Wochenbett, Station spezifische Items zur Auswahl angeboten. Einer Pflegeperson aus dem Anästhesiebereich werden andererseits zu den Themenbereichen präklinische Notfallmedizin, Anästhesie perioperativ, Schmerzbehandlung, Intensivmedizin jeweils passende spezifische Charakterisierungselemente angeboten. Auf ähnliche Weise sind folgende Verantwortungsbereiche ebenfalls subklassiert: Allgemeinmedizin, Chirurgie, Innere Medizin, Pädiatrie, Psychiatrie, Diagnostikbereiche, Apotheke, andere (Administration, Betrieb, ...). Weiter wird das Ereignis unstrukturiert beschrieben, und zwar als Fallbeschreibung bis hin zum Ereignis, ohne jene Details, die den Patienten oder den Reporter identifizieren könnten. In gleicher Weise wird getrennt davon Management \& Konsequenzen der Situation (ab Eintreten des Ereignisses) beschrieben. Die Vermeidbarkeit des Ereignisses und der Outcome werden danach wieder strukturiert erfragt.

- Die Einschätzung des Ereignisses unterscheidet in der strukturierten Erfassung die Ursache (was hat zu diesem Zwischenfall geführt) von der Bewältigungsstrategie (wichtigste/ hilfreichste Punkte beim Management).

Ereignisbeschreibung, Management und Outcomeaspekte der eingegebenen Incidents können von allen berechtigten Systembenützern eingesehen und anonym/elektronisch diskutiert werden. Unter dem Punkt Auswertung sind auch die anderen Datenelemente des Minimalen Datasets als relative Summenstatistik direkt visualisiert. Selbstverständlich haben die jeweiligen Be- 
treiber eines lokalen oder regionalen CIRSmedical-Systems vollen Zugriff auf ihre Datenbank und können entsprechend selbständige Auswertungen vornehmen.

Für geschlossene Umgebungen ist eine Windows-Applikation mit diesem Funktionsumfang frei verfügbar. Neben dem beschriebenen einfachen WEB-Reporting enthält auch diese lokale installierbare Applikation die interaktive Darstellung von rapportierten Incidents, eine eingebaute Analyse und zusätzliche Funktionen wie das INTERNET-basierte Aktualisieren der zugrundeliegenden Fachdefinitionen. Diese Stand-alone, sich selbst installierende Applikation, ist frei downloadbar mit der einzigen Vorgabe, dass die anonymen Incidents regelmässig an die zentrale, anonymisierende Sammelstelle (CIRSmedical@ unibas.ch) zwecks konsolidierter Analyse gesandt werden. Für grössere Institutionen/Spitäler mit hohem Datenverkehr und vielen verschiedenen Clients (PC, MAC, UNIX, LINUX, ...) ist die komplette WEB-Applikation zur Installation auf einem WEB-Server erhältlich.

Da die Lösung sowohl als Einzelplatz-Applikation (Windows-Umgebung) als auch als WEB-Lösung (alle Client-Betriebssysteme) zur Verfügung gestellt wird, ergeben sich folgende Einsatzformen:

I. Einsatz abteilungsintern auf einem lokalem Rechner.

II. Einsatz auf dem INTRANET eine Spitals oder Spital-Verbundes.

III. Streng geschützter Einsatz durch Closed User Groups innerhalb des Health Info Net (HIN) der FMH.

IV. Offener Einsatz auf dem INTERNET (www. CIRSmedical.org) über sichere SSL-Verbindungen zum CIRSmedical-Server oder passwortgeschützter Zugang zu einer dort definierten Closed User Group.

Das World Wide Web bietet sich als Plattform zur elektronischen Submission von IncidentReports aus folgenden Gründen an:

- Einfacher und breiter Zugang via Internet.

- Systeminduzierte Anonymität des Verfassers abhängig von der konkreten Einsatzform (vgl. Variante I.-IV. oben).

- Einheitliche Report-Strukturierung und UserFührung durch WEB-Interface.

- Direkte anonyme Feedback-Möglichkeit.

- Breite online-Visualisierung von bestehenden Incidents: Jeder kann aus Incidents von anderen lernen (präventive Wirkung).

- Anonyme Diskussionsmöglichkeit von Ereignissen.
- Zentrale Kondensierung von seltenen Ereignissen.

Verschiedene Aspekte (Herkunft der Daten, relativer Schutz gegen aussen) sprechen für die ersten beiden Einsatzformen. Breiter Zugang, rechtliche Aspekte und fachweite Abdeckung bei verbindlicheren Reporting-Absprachen sprechen eher für die dritte Variante, insbesondere da das System über den minimalen Datensatz hinaus auch das Hinzufügen von weiteren spital- oder fachspezifischen Items erlaubt (Customizing). Weitere einstellbare Möglichkeiten der zur Verfügung gestellten Software beinhalten unter anderem:

- Zugang nur nach Passwort-Eingabe.

- Separierte Benutzergruppen (eigene Passwörter).

- Manuelle Freigabe von Incidents zum Display durch lokalen Moderator/Supervisor.

- Ausblendbarkeit von «inadäquaten» Fallbeschreibungen.

- Weitere Eingabefelder zusätzlich zum minimalen Datensatz.

- Update Fach-Items via WEB-Download (XMLFile).

Die rechtliche Seite von CIRSmedical, sprich die Benutzbarkeit von CIRSmedical-Daten, in Strafprozessen ist nicht abschliessend geklärt. Zurzeit wird von verschiedenen Seiten, u. a. von Vertretern der juristischen Fakultät Basel, die Frage bearbeitet, wie eine Nichtbenutzbarkeit von CIRSmedical-Daten bei juristischen Auseinandersetzungen erreichbar wird. Grundsätzlich ist jedoch anzumerken, dass Art und Inhalt der gesammelten Daten eine Verwendung im konkreten Einzelfall ohnehin praktisch verunmöglichen, da ein Bezug der anonymen Daten zum konkreten Patienten oder Verursacher in der Regel nur schwer herzustellen ist, ausser wenn eine ganz spezifische Ereignisbeschreibung in einem System mit eingeschränktem Benutzerkreis (z. B. lokales Spitalsystem) verbunden ist.

\section{Wertung}

Critical Incident Reporting kann das Lernen aus Fehlern nachhaltig unterstützen, indem es hilft, potentiell kritische Schwachstellen aufzuzeigen. Systemveränderungen basierend auf Erkenntnissen aus einem Critical-Incident-System führen zu einer proaktiven Erhöhung der Sicherheit $[12,13]$. Das Betreiben eines solchen Systems führt implizit zu einer Veränderung der Fehler- 
kultur in dem Umfeld, in dem es betrieben wird. Eine einheitliche Beschreibung auf der Basis eines gleich strukturierten minimalen Datensatzes lässt grundsätzlich eine fach- und berufsgruppenübergreifende Analyse von rapportierten Incidents zu. Bedingt durch das erhebliche «Under-Reporting» im klinischen Alltag sind jedoch Erkenntnisse aus solchen künftigen Auswertungen mit grosser Vorsicht zu interpretieren. Der Hauptnutzen beim Betrieb solcher Critical-Incident-Systeme liegt sicher in der lokalen Beeinflussung der Fehlerkultur in dem Umfeld, in dem solche von der jeweiligen Führung unterstützten Systeme betrieben werden.

\section{Literatur}

1 Brennan TA, Leape LL, Laird NM et al. Incidence of adverse events and negligence in hospitalized patients. New Engl J Med 1991;324:370-6.

2 Leape LL, Brennan TA, Troyen A et al. The nature of adverse events in hospitalized patients: Results of the Harvard Medical Practice Study II. N Engl J Med 1991;324:377-84.

3 Wilson RM, Runciman WB, Gibberd RW et al The Quality in Australian Health Care Study. Med J Aust 1995;163:458-71.

4 Leape LL.Error in medicine. JAMA 1994;272:1851.

5 Reason JT. Human error: models and mangement BMJ 2000;320:768-70.
6 Staender S, Davies J, Helmreich B, Sexton B, Kaufmann M. The anaesthesia critical incident reporting system: an experience based database. Int J Med Inf. 1997;47:87-90.

7 Staender S, Kaufmann M, Scheidegger D: Human recoveries in the management of critical incidents in anesthesiology. Proc Am Nat Pat Assoc 1999.

8 Reason J. Managing the risks of organizational accidents. 1997. Hampshire, England, Ashgate Publishing Company.

9 Staender S, Kaufmann M, Scheidegger D. Critical Incident Reporting Systems in Anaesthesia. In: Vincent Ch, de Mol B, editors. Safety in Medicine. Oxford: Pergamon, Elsevier Science, 2000:65-82.

10 Brunner HH, Conen D, Günter P, von Gunten M, Huber F, Kehrer B, Komorowski A, Langenegger M, Scheidegger D, Schneider R, Suter P, Vincent C, Weber O. A safe healthcare system. Proposal for a national programme on patient safety improvement for Switzerland: www.swiss-q.org/ apr-2001/docs/Final_ReportE.pdf.

11 Barach P, Small SD: Reporting and preventing medical mishaps: lessons from non-medical near miss reporting systems. BMJ 2000;320:759-63.

12 Kohn LT, Corrigan JM, Donaldson MS, eds. To err is human. Building a safer health system. Washington, DC: National Academy Press, 1999.

13 Nolan TW. System changes to improve patient safety. BMJ 2000;320:771-3. 